\title{
DIRECT DETERMINATION OF THE KINEMATICS OF THE UNIVERSE AND PROPERTIES OF THE DARK ENERGY AS FUNCTIONS OF REDSHIFT
}

\author{
Ruth A. Daly \\ Department of Physics, Berks-Lehigh Valley College, Pennsylvania State University, Reading, PA 19610; rdaly@psu.edu \\ AND \\ S. G. DJorgovski \\ Division of Physics, Mathematics, and Astronomy, California Institute of Technology, \\ MS 105-24, Pasadena, CA 91125; george@astro.caltech.edu \\ Received 2004 March 29; accepted 2004 May 18
}

\begin{abstract}
Understanding the nature of dark energy, which appears to drive the expansion of the universe, is one of the central problems of physical cosmology today. In an earlier paper we proposed a novel method to determine the expansion rate $E(z)$ and the deceleration parameter $q(z)$ in a largely model-independent way, directly from the data on coordinate distances $y(z)$. Here we expand this methodology to include measurements of the pressure of dark energy $p(z)$, its normalized energy density fraction $f(z)$, and the equation-of-state parameter $w(z)$. We then apply this methodology to a new, combined data set of distances to supernovae and radio galaxies. In evaluating $E(z)$ and $q(z)$, we make only the assumptions that the FRW metric applies and that the universe is spatially flat (an assumption strongly supported by modern cosmic microwave background radiation measurements). The determinations of $E(z)$ and $q(z)$ are independent of any theory of gravity. For evaluations of $p(z), f(z)$, and $w(z)$, a theory of gravity must be adopted, and general relativity is assumed here. No a priori assumptions regarding the properties or redshift evolution of the dark energy are needed. We obtain trends for $y(z)$ and $E(z)$ that are fully consistent with the standard Friedmann-Lemaître concordance cosmology with $\Omega_{0}=0.3$ and $\Lambda_{0}=0.7$. The measured trend for $q(z)$ deviates systematically from the predictions of this model on a $\sim 1-2 \sigma$ level but may be consistent for smaller values of $\Lambda_{0}$. We confirm our previous result that the universe transitions from acceleration to deceleration at a redshift $z_{T} \approx 0.4$. The trends for $p(z), f(z)$, and $w(z)$ are consistent with being constant at least out to $z \sim 0.3-0.5$ and broadly consistent with being constant out to higher redshifts, but with large uncertainties. For the present values of these parameters we obtain $E_{0}=0.97 \pm 0.03, q_{0}=-0.35 \pm 0.15$, $p_{0}=-0.6 \pm 0.15, f_{0}=-0.62-\left(\Omega_{0}-0.3\right) \pm 0.05$, and $w_{0}=-0.9-\epsilon\left(\Omega_{0}-0.3\right) \pm 0.1$, where $\Omega_{0}$ is the density parameter for nonrelativistic matter and $\epsilon \approx 1.5 \pm 0.1$. We note that in the standard Friedmann-Lemaitre models $p_{0}=-\Lambda_{0}$, and thus we can measure the value of the cosmological constant directly and obtain results in agreement with other contemporary results.
\end{abstract}

Subject headings: cosmological parameters — cosmology: observations — cosmology: theory — dark matter - equation of state

On-line material: machine-readable table

\section{INTRODUCTION}

Observations of supernovae (SNe; Riess et al. 1998, 2004; Perlmutter et al. 1999; Tonry et al. 2003; Knop et al. 2003; Barris et al. 2004) indicate that the universe is accelerating in its expansion. Precision measurements of cosmological parameters from cosmic microwave background radiation (CMBR) experiments confirm this remarkable finding (e.g., Bennett et al. 2003; Spergel et al. 2003 and references therein). Results similar to those obtained using $\mathrm{SNe}$ are also obtained using radio galaxies (RGs; Guerra \& Daly 1998; Guerra et al. 2000; Daly \& Guerra 2002; Podariu et al. 2003). The acceleration of the universe at the present epoch is one of the key results of modern cosmology, with potentially significant implications for fundamental physics as well. The nature of the "dark energy," which apparently drives the cosmic acceleration, is unknown, and it is crucially important to extract information about it from the data in a manner that is as direct and modelindependent as possible.

In Daly \& Djorgovski (2003, hereafter Paper I), we showed how the data could be used to study the dimensionless expansion rate of the universe $E(z)$ and the deceleration parameter of the universe $q(z)$ directly from combinations of the first and second derivatives of the coordinate distance. These determinations only depend on the Friedmann-Robertson-Walker (FRW) metric and an assumption of spatially flat geometry, which is now very well established by the CMBR experiments. The evaluations do not require the specification of anything else, including a theory of gravity, and thus are direct and model-independent.

The use of model-independent methods to derive information about the dark energy are also discussed, for example, by Huterer \& Turner (1999, 2001), Saini et al. (2000), Tegmark (2002), Sahni et al. (2003), Huterer \& Starkman (2003), Wang \& Freese (2004), Wang \& Tegmark (2004), Wang et al. (2004), and Daly \& Djorgovski (2004). The work of Huterer \& Turner focuses on determinations of $w(z)$, as does that of Huterer \& Starkman (2003). Wang \& Freese (2004) focus on the determination of the energy density of the dark energy and use an approach that is complementary to that used here, by integrating over shells in redshift space to obtain the energy density as a function of redshift, while we differentiate the 
data to obtain this function. The approach taken by most authors to extract the redshift behavior of the dark energy is to integrate over an assumed functional form of the redshift evolution of the dark energy, having first adopted a theory of gravity (e.g., Starobinsky 1988; Huterer \& Turner 1999, 2001; Saini et al. 2000; Chiba \& Nakamura 2000; Maor et al. 2001; Golaith et al. 2001; Wang \& Garnavich 2001; Astier 2001; Gerke \& Efstathiou 2002; Weller \& Albrecht 2002; Padmanabhan \& Choudhury 2003; Tegmark 2002; Huterer \& Starkman 2003; Sahni et al. 2003; Alam et al. 2003, 2004; Wang \& Freese 2004; Wang et al. 2004; Wang \& Tegmark 2004; Nessier \& Perivolaropoulos 2004; Gong 2004; Zhu et al. 2004; Elgaroy \& Multamaki 2004; Huterer \& Cooray 2004). However, it can be difficult to extract information about the redshift behavior of the dark energy using these "integral" approaches (Maor et al. 2001; Barger \& Marfatia 2001). Thus, we continue to follow the complementary approach of differentiating the data, as described in Paper I.

Here the approach presented in Paper I is taken a step further to obtain the pressure, energy density, and equation of state of the dark energy directly from combinations of the first and second derivatives of the coordinate distance with respect to redshift. This approach is complementary to the standard approach of assuming a theory of gravity, assuming a parameterization for the dark energy and its redshift evolution, and obtaining the best-fit model parameters.

We apply this methodology to an improved set of distances to SNe from Riess et al. (2004), supplemented with the data on high-redshift RGs from Paper I.

\section{THEORY}

This work builds on Paper I, and we refer the reader to it for more details and discussion. It is well known that the dimensionless expansion rate $E(z)$ can be written as the derivative of the dimensionless coordinate distances $y(z)$ (e.g., Weinberg 1972; Peebles 1993; Peebles \& Ratra 2003); the expression is particularly simple when the space curvature term is equal to zero. In this case,

$$
\left(\frac{\dot{a}}{a}\right) H_{0}^{-1} \equiv E(z)=\left(\frac{d y}{d z}\right)^{-1},
$$

where $a$ is the cosmic scale factor and $H_{0}=\left.(\dot{a} / a)\right|_{0}$ evaluated at a redshift of zero is the Hubble constant. This representation follows directly from the FRW line element and does not require the use of a theory of gravity. Similarly, it was shown in Paper I that the dimensionless deceleration parameter

$$
-\left(\frac{\ddot{a} a}{\dot{a}^{2}}\right) \equiv q(z)=-\left[1+(1+z)\left(\frac{d y}{d z}\right)^{-1} \frac{d^{2} y}{d z^{2}}\right]
$$

also follows directly from the FRW line element and does not rely on a theory of gravity. Thus, measurements of the dimensionless coordinate distance to sources at different redshifts can be used to determine $d y / d z$ and $d^{2} y / d z^{2}$, which can then be used to determine $E(z)$ and $q(z)$.

In addition, if a theory of gravity is specified, the measurements of $d y / d z$ and $d^{2} y / d z^{2}$ can be used to determine the pressure, energy density, and equation of state of the dark energy as functions of redshift. Thus, we can use the data to determine these functions directly, which provides an approach that is complementary to the standard one of assuming a functional form a priori and then fitting the parameters of the chosen function. To determine the pressure, energy density, and equation of state of the dark energy as functions of redshift, the theory of gravity adopted is general relativity.

In a spatially flat, homogeneous, isotropic universe with nonrelativistic matter and dark energy, Einstein's equations are

$$
\begin{gathered}
\frac{\ddot{a}}{a}=-\frac{4 \pi G}{3}\left(\rho_{m}+\rho_{\mathrm{DE}}+3 P_{\mathrm{DE}}\right), \\
\left(\frac{\dot{a}}{a}\right)^{2}=\frac{8 \pi G}{3}\left(\rho_{m}+\rho_{\mathrm{DE}}\right),
\end{gathered}
$$

where $\rho_{m}$ is the mean mass-energy density of nonrelativistic matter, $\rho_{\mathrm{DE}}$ is the mean mass-energy density of the dark energy, and $P_{\mathrm{DE}}$ is the pressure of the dark energy. Combining these equations, we find $\ddot{a} / a=-0.5\left[(\dot{a} / a)^{2}+(8 \pi G) P_{\mathrm{DE}}\right]$.

Using the standard definition of the critical density at the present epoch $\rho_{0 c}=3 H_{0}^{2} /(8 \pi G)$, it is easy to show that

$$
p(z) \equiv \frac{P_{\mathrm{DE}}(z)}{\rho_{0 c}}=\frac{E^{2}(z)}{3}[2 q(z)-1] .
$$

Equations (1) and (2) can be used to obtain the pressure of the dark energy as a function of redshift

$$
p(z)=-\left(\frac{d y}{d z}\right)^{-2}\left[1+\frac{2}{3}(1+z)\left(\frac{d y}{d z}\right)^{-1} \frac{d^{2} y}{d z^{2}}\right]
$$

Thus, the pressure of the dark energy can be determined directly from measurements under the same assumptions as above. Moreover, for the standard Friedmann-Lemaître models, it can be shown that $p=-\Omega_{\Lambda}$, giving us a way to measure the value of the cosmological constant directly.

Similarly, the energy density of the dark energy can be obtained directly from the data using equations (1) and (4):

$$
f(z) \equiv \frac{\rho_{\mathrm{DE}}(z)}{\rho_{0 c}}=\left(\frac{d y}{d z}\right)^{-2}-\Omega_{0}(1+z)^{3},
$$

where $\Omega_{0}=\rho_{0 m} / \rho_{0 c}$ is the fractional contribution of nonrelativistic matter to the total critical density at zero redshift, and it is assumed that this nonrelativistic matter evolves as $(1+z)^{3}$.

The equation of state parameter $w(z)$ is defined to be the ratio of the pressure of the dark energy to its energy density $w(z) \equiv P_{\mathrm{DE}}(z) / \rho_{\mathrm{DE}}(z)$. Combining equations (6) and (7), it is easy to show that

$$
w(z)=\frac{-\left[1+(2 / 3)(1+z)(d y / d z)^{-1}\left(d^{2} y / d z^{2}\right)\right]}{1-(d y / d z)^{2} \Omega_{0}(1+z)^{3}} .
$$

\section{DATA ANALYSIS AND RESULTS}

Our method is based on a robust numerical differentiation of data on coordinate distances $y(z)$, which is described in detail in Paper I. One of the advantages of our method is that distances from different types of measurements (e.g., SN standard candles and RG standard rulers) can be combined, separating the astrophysical questions (how standard are these sources, what are the selection effects, etc.) from analyses dealing with pure geometry and kinematics. 
TABLE 1

Radio Galaxy Dimensionless Coordinate Distances

\begin{tabular}{|c|c|c|c|}
\hline Source & Redshift & $y$ & $\sigma(y)$ \\
\hline $3 \mathrm{C} 405 \ldots .$. & 0.0560 & 0.0556 & 0.0095 \\
\hline 3C $244.1 \ldots \ldots \ldots$ & 0.4300 & 0.4559 & 0.0700 \\
\hline 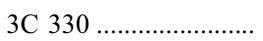 & 0.5490 & 0.4019 & 0.0637 \\
\hline 3C 427.1 & 0.5720 & 0.3193 & 0.0488 \\
\hline 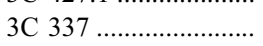 & 0.6300 & 0.6094 & 0.0687 \\
\hline 3C 55 & 0.7200 & 0.5986 & 0.0678 \\
\hline 3C $247 \ldots \ldots \ldots$ & 0.7490 & 0.6255 & 0.0665 \\
\hline 3C $265 \ldots \ldots \ldots$ & 0.8110 & 0.6757 & 0.0787 \\
\hline 3C $325 \ldots \ldots \ldots$ & 0.8600 & 0.8180 & 0.1489 \\
\hline 3C $289 \ldots \ldots \ldots \ldots$ & 0.9670 & 0.6809 & 0.1030 \\
\hline 3C $268.1 \ldots \ldots \ldots \ldots \ldots$ & 0.9740 & 0.7679 & 0.1186 \\
\hline 3C $280 \ldots \ldots \ldots$ & 0.9960 & 0.7108 & 0.1073 \\
\hline 3C $356 \ldots \ldots \ldots \ldots$ & 1.0790 & 0.8284 & 0.1421 \\
\hline 3C $267 \ldots \ldots \ldots$ & 1.1440 & 0.7526 & 0.1206 \\
\hline 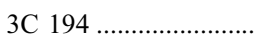 & 1.1900 & 1.1412 & 0.1975 \\
\hline 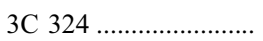 & 1.2100 & 0.9730 & 0.2350 \\
\hline 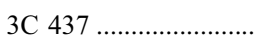 & 1.4800 & 0.8211 & 0.1895 \\
\hline $3 \mathrm{C} 68.2 \ldots$ & 1.5750 & 1.4770 & 0.3690 \\
\hline 3C $322 \ldots \ldots \ldots \ldots \ldots \ldots \ldots$ & 1.6810 & 1.1406 & 0.2309 \\
\hline $3 \mathrm{C} 239$ & 1.7900 & 1.2144 & 0.2376 \\
\hline
\end{tabular}

Two data samples are included in this study: the RG sample presented and described by Guerra et al. (2000), Daly \& Guerra (2002), Podariu et al. (2003), and in Paper I, and the latest cosmological SN sample from Riess et al. (2004). The RG sample consists of 20 RGs with redshifts between zero and 1.8 (Guerra et al. 2000). The SN sample that we use here consists of the "gold" SNe, with redshifts between zero and 1.7 (Riess et al. 2004). We refer to the original papers for the description of the measurements and other pertinent information.

The dimensionless coordinate distances $y(z)$ to RGs were determined in Paper I for normalizations obtained using RGs alone (referred to as $y_{s}$ ) and using a joint sample of RGs and $\mathrm{SNe}$ (referred to as $y_{j}$ ). The current $\mathrm{SN}$ sample is used to obtain new values of $y_{j}$ by using them to determine a new normalization for the RGs, and these are listed in Table 1. These values are nearly identical to those in Paper I.

The dimensionless coordinate distances to $\mathrm{SNe}$ are listed in Table 2. To determine these from the distance moduli published by Riess et al. (2004), the value of $H_{0}$ adopted by Riess et al. (2004) must be known. This was not given explicitly in the Riess et al. paper, as it was not needed for their analysis. Since we essentially need to remove the value and uncertainty of $H_{0}$ put in by Riess et al. (2004), we determine the effective value of $H_{0}$ applicable to that $\mathrm{SN}$ sample by using the subsample of SNe with $z<0.1$, where the expansion must be close to linear and the Hubble relation $H_{0}=v(1+z) / d_{L}$ is valid. Using the luminosity distance $d_{L}$ obtained directly from the distance moduli tabulated by Riess et al. (2004), we get $H_{0}=66.4 \pm 0.8 \mathrm{~km} \mathrm{~s}^{-1} \mathrm{Mpc}^{-1}$. This value is used simply to obtain the dimensionless coordinate distances $y(z)$ from the published luminosity distances using the relation $y(z)=$ $\left(H_{0} / c\right) d_{L}(1+z)^{-1}$, but it does not affect our analysis in any other way. It is not meant as a measurement of $H_{0}$ per se, but just as an internally consistent scaling factor, and the error quoted above is just statistical and does not include any other components due to calibrations, etc. The values of $y(z)$ given in Tables 1 and 2 can then be easily converted to distances in parsecs for any desired value of $H_{0}$.
We test for the consistency between the distance measurements from $\mathrm{SNe}$ and RGs in the redshift interval where they overlap (Fig. 1). Reassuringly, we find no significant systematic offset, which indicates that the joint sample is sufficiently homogeneous for our purposes. We note that we repeated our analysis for the SN sample alone and got essentially the same results, but with larger error bars at the highredshift end, where the sample of SNe is still very sparse and RGs provide valuable supplementary data. At the low redshifts, SNe dominate the results.

Our methodology is described in detail in Paper I, which also includes extensive tests using simulated data. To summarize briefly, we perform a statistically robust numerical differentiation of the $y(z)$ data, in order to obtain the first and second derivatives, $d y / d z$ and $d^{2} y / d z^{2}$, used in equations (1)-(8). While differentiation of noisy and sparse data is generally inadvisable, it is possible and may be useful if one keeps careful track of the errors and other limitations posed by the data.

The procedure is based on properly weighted second-order least-squares fits at a closely spaced grid of redshift points in a sliding redshift window, which is generally chosen to be sufficiently large ( $\Delta z=0.4$ or 0.6$)$ to have enough data points for meaningful measurements of the three fit coefficients. The fit coefficients and their errors essentially correspond to the best-fit values for $y(z), d y / d z$, and $d^{2} y / d z^{2}$. We are effectively doing a Taylor series expansion for the expansion law as a function of redshift. Statistical errors, including all covariance terms, are propagated in the standard manner. While the large values of $\Delta z$ are needed in order to obtain stable fits, that also means that there are very few independent intervals: we are essentially mapping the trends rather than to trying to bin the data. We find that the derived mean trends for all quantities of interest described below do not depend significantly on the value of $\Delta z$ used, i.e., that the results are robust with respect to this parameter. However, the statistical errors increase dramatically for lower values of $\Delta z$ because of the smaller numbers of enclosed data points.

While the fitting procedure generates statistically rigorous errors at every point, that does not include any effects of the uneven data sampling and sample variance (see the discussion in Paper I). The $1 \sigma$ error intervals plotted in the figures reflect only the statistical errors. The apparent "bumps and wiggles" are presumably indicative of the sparse sampling, especially at higher redshifts. Any systematic errors in $y(z)$ measurements that may be present in the data are also absorbed there. Thus, one should not believe any such features in the plots, but only look at the global trends. We also regard the values for all derived quantities at lower redshifts to be fairly reliable, since the data are best and the sampling is densest as $z \rightarrow 0$.

As in Paper I, we perform a test of the procedure using a simulated data set that mimics the anticipated SN measurements from the $S N A P /$ JDEM satellite $^{1}$ with a known assumed cosmology, namely the standard Friedmann-Lemaittre model with $\Omega_{0}=0.3$ and $\Lambda_{0}=0.7$ (see Paper I for more details on this simulated data set). The results for the dark energy parameters as functions of redshift are shown in Figure 2. We see that our method can recover robustly the assumed parameters, at least out to $z \approx 0.9$. Reassured by this test, we turn to the analysis of actual data.

We do not endeavor here to examine or advocate the primary measurements of distances we use in our analysis; that

\footnotetext{
${ }^{1}$ See http://snap.lbl.gov.
} 
TABLE 2

SN Ia Dimensionless Coordinate Distances

\begin{tabular}{|c|c|c|c|c|}
\hline Source & Redshift & $y$ & $\sigma(y)$ & Sample \\
\hline $1990 \mathrm{~T} \ldots \ldots$. & 0.040 & 0.040 & 0.0035 & Gold \\
\hline 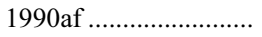 & 0.050 & 0.049 & 0.0048 & Gold \\
\hline $19900 \ldots$ & 0.031 & 0.033 & 0.0030 & Gold \\
\hline 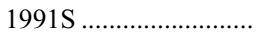 & 0.056 & 0.061 & 0.0050 & Gold \\
\hline $1991 U \ldots \ldots$. & 0.033 & 0.027 & 0.0025 & Gold \\
\hline 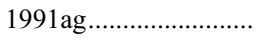 & 0.014 & 0.015 & 0.0017 & Gold \\
\hline 1992J .. & 0.046 & 0.039 & 0.0038 & Gold \\
\hline $1992 \mathrm{P} \ldots \ldots .$. & 0.027 & 0.029 & 0.0027 & Gold \\
\hline 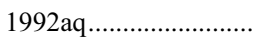 & 0.101 & 0.112 & 0.0103 & Gold \\
\hline 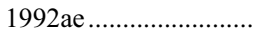 & 0.075 & 0.074 & 0.0065 & Gold \\
\hline 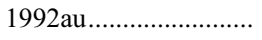 & 0.061 & 0.060 & 0.0061 & Gold \\
\hline 1992al..... & 0.014 & 0.015 & 0.0017 & Gold \\
\hline $1992 \mathrm{ag} . . . \ldots . .$. & 0.026 & 0.022 & 0.0025 & Gold \\
\hline $1992 \mathrm{bl} \mathrm{\ldots .......}$ & 0.043 & 0.043 & 0.0038 & Gold \\
\hline 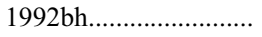 & 0.045 & 0.052 & 0.0043 & Gold \\
\hline $1992 b g \ldots .$. & 0.036 & 0.037 & 0.0032 & Gold \\
\hline 1992bk................ & 0.058 & 0.056 & 0.0049 & Gold \\
\hline 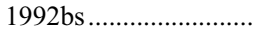 & 0.063 & 0.071 & 0.0062 & Gold \\
\hline 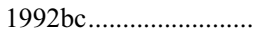 & 0.019 & 0.021 & 0.0022 & Gold \\
\hline 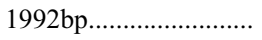 & 0.079 & 0.079 & 0.0066 & Gold \\
\hline 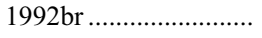 & 0.088 & 0.084 & 0.0108 & Gold \\
\hline 1992bo.......................... & 0.018 & 0.019 & 0.0020 & Gold \\
\hline 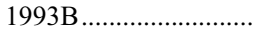 & 0.071 & 0.074 & 0.0065 & Gold \\
\hline 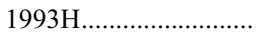 & 0.025 & 0.023 & 0.0022 & Gold \\
\hline 19930......... & 0.052 & 0.057 & 0.0047 & Gold \\
\hline 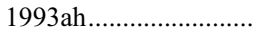 & 0.029 & 0.027 & 0.0027 & Gold \\
\hline $1993 \mathrm{ac}$ & 0.049 & 0.051 & 0.0047 & Gold \\
\hline 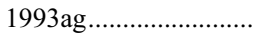 & 0.050 & 0.055 & 0.0048 & Gold \\
\hline 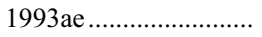 & 0.018 & 0.016 & 0.0017 & Gold \\
\hline $1994 \mathrm{~B} \ldots \ldots$. & 0.089 & 0.102 & 0.0080 & Silver \\
\hline 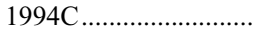 & 0.051 & 0.045 & 0.0033 & Silver \\
\hline $1994 \mathrm{M} \ldots \ldots .$. & 0.024 & 0.023 & 0.0021 & Gold \\
\hline 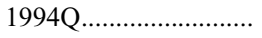 & 0.029 & 0.030 & 0.0026 & Gold \\
\hline 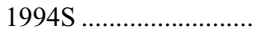 & 0.016 & 0.017 & 0.0019 & Gold \\
\hline 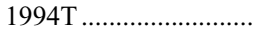 & 0.036 & 0.034 & 0.0031 & Gold \\
\hline 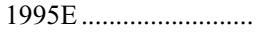 & 0.012 & 0.009 & 0.0011 & Silver \\
\hline 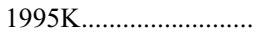 & 0.478 & 0.469 & 0.0497 & Gold \\
\hline $1995 \mathrm{M} \ldots \ldots \ldots$ & 0.053 & 0.057 & 0.0039 & Silver \\
\hline 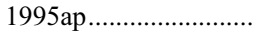 & 0.230 & 0.220 & 0.0467 & Silver \\
\hline 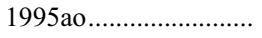 & 0.300 & 0.242 & 0.0668 & Silver \\
\hline $1995 \mathrm{ae}$ & 0.067 & 0.067 & 0.0105 & Silver \\
\hline 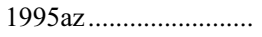 & 0.450 & 0.407 & 0.0394 & Gold \\
\hline 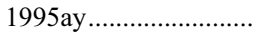 & 0.480 & 0.445 & 0.0410 & Gold \\
\hline 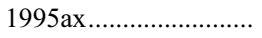 & 0.615 & 0.509 & 0.0539 & Gold \\
\hline 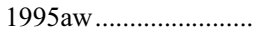 & 0.400 & 0.405 & 0.0354 & Gold \\
\hline 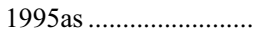 & 0.498 & 0.648 & 0.0716 & Silver \\
\hline 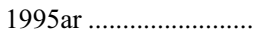 & 0.465 & 0.551 & 0.0558 & Silver \\
\hline $1995 \mathrm{ac}$ & 0.049 & 0.042 & 0.0039 & Gold \\
\hline 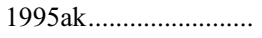 & 0.022 & 0.019 & 0.0019 & Gold \\
\hline 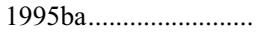 & 0.388 & 0.414 & 0.0362 & Gold \\
\hline 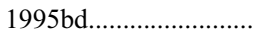 & 0.015 & 0.014 & 0.0017 & Gold \\
\hline $1996 \mathrm{C} \ldots \ldots . . .$. & 0.028 & 0.033 & 0.0030 & Gold \\
\hline 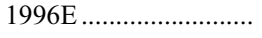 & 0.425 & 0.340 & 0.0626 & Gold \\
\hline 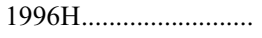 & 0.620 & 0.572 & 0.0790 & Gold \\
\hline 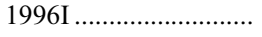 & 0.570 & 0.514 & 0.0592 & Gold \\
\hline 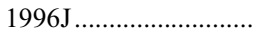 & 0.300 & 0.271 & 0.0312 & Gold \\
\hline 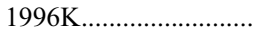 & 0.380 & 0.407 & 0.0412 & Gold \\
\hline 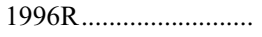 & 0.160 & 0.125 & 0.0230 & Silver \\
\hline 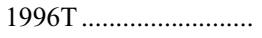 & 0.240 & 0.244 & 0.0483 & Silver \\
\hline 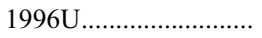 & 0.430 & 0.453 & 0.0709 & Gold \\
\hline 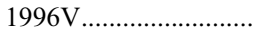 & 0.025 & 0.025 & 0.0029 & Silver \\
\hline 1996ab......................... & 0.124 & 0.136 & 0.0138 & Gold \\
\hline 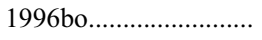 & 0.017 & 0.013 & 0.0016 & Gold \\
\hline 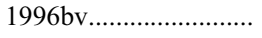 & 0.017 & 0.015 & 0.0016 & Gold \\
\hline $1996 b 1 \ldots . .$. & 0.035 & 0.037 & 0.0032 & Gold \\
\hline
\end{tabular}

TABLE 2-Continued

\begin{tabular}{|c|c|c|c|c|}
\hline Source & Redshift & $y$ & $\sigma(y)$ & Sample \\
\hline $1996 \mathrm{cg} \ldots$ & 0.490 & 0.487 & 0.0426 & Silver \\
\hline $1996 \mathrm{~cm} . . . \ldots \ldots \ldots \ldots . . . . .$. & 0.450 & 0.501 & 0.0438 & Silver \\
\hline 1996cl....................... & 0.828 & 0.750 & 0.1589 & Gold \\
\hline 1996сі..................... & 0.495 & 0.417 & 0.0365 & Gold \\
\hline $1996 \mathrm{cf}$..................... & 0.570 & 0.505 & 0.0442 & Silver \\
\hline 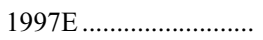 & 0.013 & 0.014 & 0.0017 & Gold \\
\hline 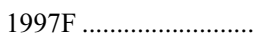 & 0.580 & 0.568 & 0.0549 & Gold \\
\hline 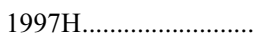 & 0.526 & 0.472 & 0.0391 & Gold \\
\hline 1997I ............................. & 0.172 & 0.171 & 0.0142 & Gold \\
\hline 1997N …….................... & 0.180 & 0.186 & 0.0154 & Gold \\
\hline 1997P ……..................... & 0.472 & 0.467 & 0.0408 & Gold \\
\hline 1997Q …….................... & 0.430 & 0.387 & 0.0321 & Gold \\
\hline 1997R ……................. & 0.657 & 0.602 & 0.0555 & Gold \\
\hline $1997 Y \ldots$ & 0.017 & 0.018 & 0.0019 & Gold \\
\hline 1997ai.............................. & 0.450 & 0.401 & 0.0425 & Gold \\
\hline $1997 \mathrm{ac}$ & 0.320 & 0.327 & 0.0271 & Gold \\
\hline 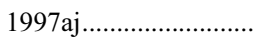 & 0.581 & 0.470 & 0.0411 & Gold \\
\hline 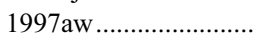 & 0.440 & 0.502 & 0.0925 & Gold \\
\hline 1997as ................... & 0.508 & 0.312 & 0.0503 & Gold \\
\hline 1997am ........................ & 0.416 & 0.411 & 0.0360 & Gold \\
\hline 1997ар.................. & 0.830 & 0.712 & 0.0623 & Gold \\
\hline 1997af .................... & 0.579 & 0.523 & 0.0458 & Gold \\
\hline 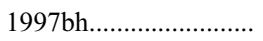 & 0.420 & 0.351 & 0.0371 & Gold \\
\hline 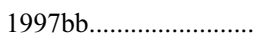 & 0.518 & 0.537 & 0.0742 & Gold \\
\hline 1997bj ....................... & 0.334 & 0.253 & 0.0350 & Gold \\
\hline 1997ck ...................... & 0.970 & 0.753 & 0.1317 & Silver \\
\hline $1997 \mathrm{cn}$ & 0.018 & 0.017 & 0.0020 & Gold \\
\hline 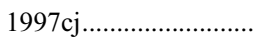 & 0.500 & 0.521 & 0.0480 & Gold \\
\hline 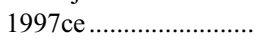 & 0.440 & 0.401 & 0.0350 & Gold \\
\hline 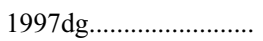 & 0.030 & 0.036 & 0.0033 & Gold \\
\hline 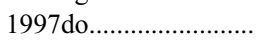 & 0.010 & 0.012 & 0.0019 & Gold \\
\hline 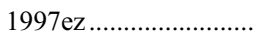 & 0.778 & 0.720 & 0.1160 & Gold \\
\hline 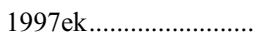 & 0.860 & 0.761 & 0.1052 & Gold \\
\hline 1997eq......................... & 0.538 & 0.490 & 0.0406 & Gold \\
\hline $1997 \mathrm{ff}$......................... & 1.755 & 1.025 & 0.1653 & Gold \\
\hline 1998I ........................ & 0.886 & 0.448 & 0.1672 & Gold \\
\hline 1998J ....................... & 0.828 & 0.638 & 0.1793 & Gold \\
\hline 1998М ………................ & 0.630 & 0.454 & 0.0502 & Gold \\
\hline 1998V & 0.017 & 0.017 & 0.0018 & Gold \\
\hline 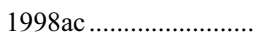 & 0.460 & 0.352 & 0.0649 & Gold \\
\hline 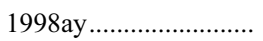 & 0.638 & 0.618 & 0.1024 & Silver \\
\hline 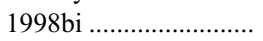 & 0.740 & 0.595 & 0.0822 & Gold \\
\hline 1998be …….......... & 0.644 & 0.484 & 0.0580 & Silver \\
\hline 1998ba..................... & 0.430 & 0.459 & 0.0528 & Gold \\
\hline 1998bp & 0.010 & 0.010 & 0.0014 & Gold \\
\hline 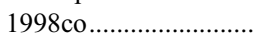 & 0.017 & 0.019 & 0.0021 & Gold \\
\hline $1998 \mathrm{cs}$ & 0.033 & 0.035 & 0.0031 & Gold \\
\hline 1998dx........................ & 0.053 & 0.052 & 0.0043 & Gold \\
\hline 1998ef ............................ & 0.017 & 0.015 & 0.0016 & Gold \\
\hline 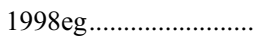 & 0.023 & 0.026 & 0.0024 & Gold \\
\hline 1999Q & 0.460 & 0.493 & 0.0613 & Gold \\
\hline 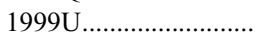 & 0.500 & 0.524 & 0.0458 & Gold \\
\hline 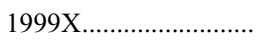 & 0.026 & 0.026 & 0.0024 & Gold \\
\hline 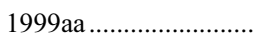 & 0.016 & 0.018 & 0.0020 & Gold \\
\hline 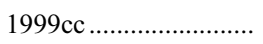 & 0.032 & 0.032 & 0.0028 & Gold \\
\hline $1999 \mathrm{cp}$ & 0.010 & 0.011 & 0.0016 & Gold \\
\hline 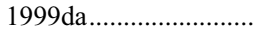 & 0.012 & 0.014 & 0.0021 & Silver \\
\hline 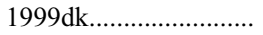 & 0.014 & 0.017 & 0.0020 & Gold \\
\hline 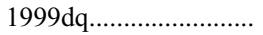 & 0.014 & 0.012 & 0.0015 & Gold \\
\hline $1999 \mathrm{ef}$ & 0.038 & 0.046 & 0.0038 & Gold \\
\hline $1999 \mathrm{fw}$ & 0.278 & 0.274 & 0.0518 & Gold \\
\hline 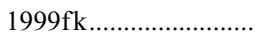 & 1.056 & 0.762 & 0.0807 & Gold \\
\hline $1999 \mathrm{fm}$ & 0.949 & 0.713 & 0.0821 & Gold \\
\hline 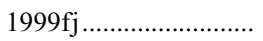 & 0.815 & 0.689 & 0.1047 & Gold \\
\hline 1999ff ...................... & 0.455 & 0.437 & 0.0563 & Gold \\
\hline $1999 \mathrm{fv}$ & 1.190 & 0.696 & 0.1090 & Gold \\
\hline
\end{tabular}


TABLE 2-Continued

\begin{tabular}{|c|c|c|c|c|}
\hline Source & Redshift & $y$ & $\sigma(y)$ & Sample \\
\hline $1999 \mathrm{fh} . . . . . . . .$. & 0.369 & 0.341 & 0.0487 & Silver \\
\hline 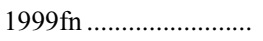 & 0.477 & 0.448 & 0.0434 & Gold \\
\hline 1999gp & 0.026 & 0.029 & 0.0026 & Gold \\
\hline 2000B & 0.019 & 0.018 & 0.0019 & Gold \\
\hline 2000bk ........................... & 0.027 & 0.025 & 0.0025 & Gold \\
\hline $2000 \mathrm{cf}$ & 0.036 & 0.041 & 0.0034 & Gold \\
\hline $2000 \mathrm{cn}$ & 0.023 & 0.023 & 0.0022 & Gold \\
\hline 2000ce ............................ & 0.016 & 0.017 & 0.0018 & Silver \\
\hline $2000 \mathrm{dk}$ & 0.016 & 0.017 & 0.0018 & Gold \\
\hline 2000dz ............................. & 0.500 & 0.524 & 0.0579 & Gold \\
\hline 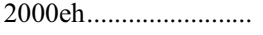 & 0.490 & 0.451 & 0.0519 & Gold \\
\hline 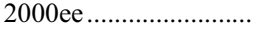 & 0.470 & 0.532 & 0.0563 & Gold \\
\hline 2000eg.............................. & 0.540 & 0.354 & 0.0669 & Gold \\
\hline 2000ea ............................... & 0.420 & 0.224 & 0.0330 & Silver \\
\hline $2000 \mathrm{ec}$ & 0.470 & 0.539 & 0.0521 & Gold \\
\hline $2000 \mathrm{fr}$ & 0.543 & 0.493 & 0.0431 & Gold \\
\hline $2000 \mathrm{fa}$ & 0.022 & 0.022 & 0.0022 & Gold \\
\hline $2001 V$ & 0.016 & 0.015 & 0.0015 & Gold \\
\hline $2001 \mathrm{fs}$ & 0.873 & 0.665 & 0.1163 & Gold \\
\hline 2001 fo ............................. & 0.771 & 0.526 & 0.0412 & Gold \\
\hline 2001hy.............................. & 0.811 & 0.761 & 0.1226 & Gold \\
\hline $2001 \mathrm{hx}$ & 0.798 & 0.735 & 0.1049 & Gold \\
\hline $2001 \mathrm{hs}$ & 0.832 & 0.620 & 0.0827 & Gold \\
\hline 2001hu.............................. & 0.882 & 0.709 & 0.0979 & Gold \\
\hline 2001iw.............................. & 0.340 & 0.229 & 0.0285 & Gold \\
\hline 2001iv ............................. & 0.397 & 0.239 & 0.0330 & Gold \\
\hline 2001iy ............................ & 0.570 & 0.531 & 0.0758 & Gold \\
\hline 2001ix ........................... & 0.710 & 0.527 & 0.0777 & Gold \\
\hline 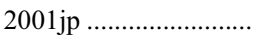 & 0.528 & 0.519 & 0.0597 & Gold \\
\hline 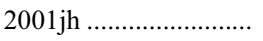 & 0.884 & 0.824 & 0.0721 & Gold \\
\hline $2001 \mathrm{jb}$ & 0.698 & 0.604 & 0.0890 & Silver \\
\hline 2001 jf ............................ & 0.815 & 0.802 & 0.1034 & Gold \\
\hline 2001jm ........................... & 0.977 & 0.678 & 0.0811 & Gold \\
\hline 2001kd............................ & 0.935 & 0.718 & 0.1257 & Silver \\
\hline 2002P …........................ & 0.719 & 0.567 & 0.0679 & Silver \\
\hline 2002ab.............................. & 0.422 & 0.395 & 0.0309 & Silver \\
\hline 2002ad............................. & 0.514 & 0.439 & 0.0546 & Silver \\
\hline $2002 d c$ & 0.475 & 0.402 & 0.0352 & Gold \\
\hline 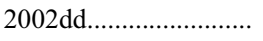 & 0.950 & 0.736 & 0.0882 & Gold \\
\hline $2002 \mathrm{fw}$ & 1.300 & 1.090 & 0.0953 & Gold \\
\hline $2002 f x$ & 1.400 & 0.961 & 0.1992 & Silver \\
\hline $2002 \mathrm{hr}$ & 0.526 & 0.580 & 0.0721 & Gold \\
\hline $2002 \mathrm{hp}$ & 1.305 & 0.836 & 0.0847 & Gold \\
\hline $2002 \mathrm{kc}$ & 0.216 & 0.212 & 0.0176 & Silver \\
\hline 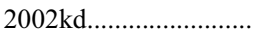 & 0.735 & 0.529 & 0.0463 & Gold \\
\hline 2002ki ............................ & 1.140 & 0.961 & 0.1327 & Gold \\
\hline 2003az ............................. & 1.265 & 1.071 & 0.0987 & Gold \\
\hline 2003ak ............................... & 1.551 & 0.996 & 0.1009 & Gold \\
\hline 2003bd.............................. & 0.670 & 0.576 & 0.0743 & Gold \\
\hline 2003be ............................ & 0.640 & 0.555 & 0.0537 & Gold \\
\hline 2003 dy .............................. & 1.340 & 0.968 & 0.1114 & Gold \\
\hline 2003es .......................... & 0.954 & 0.813 & 0.1161 & Gold \\
\hline 2003 eq & 0.839 & 0.712 & 0.0721 & Gold \\
\hline $2003 \mathrm{eb}$ & 0.899 & 0.623 & 0.0717 & Gold \\
\hline 20031v ……....................... & 0.940 & 0.678 & 0.0624 & Gold \\
\hline
\end{tabular}

Note.-Table 2 is also available in machine-readable form in the electronic edition of the Astrophysical Journal.

was done in the original papers from which they came. Our purpose here is to illustrate the methodology and seek some early hints about the possible cosmological trends in the data, assuming that the data are sound. Better and larger data sets in the future can be explored using this methodology with a much greater potential.

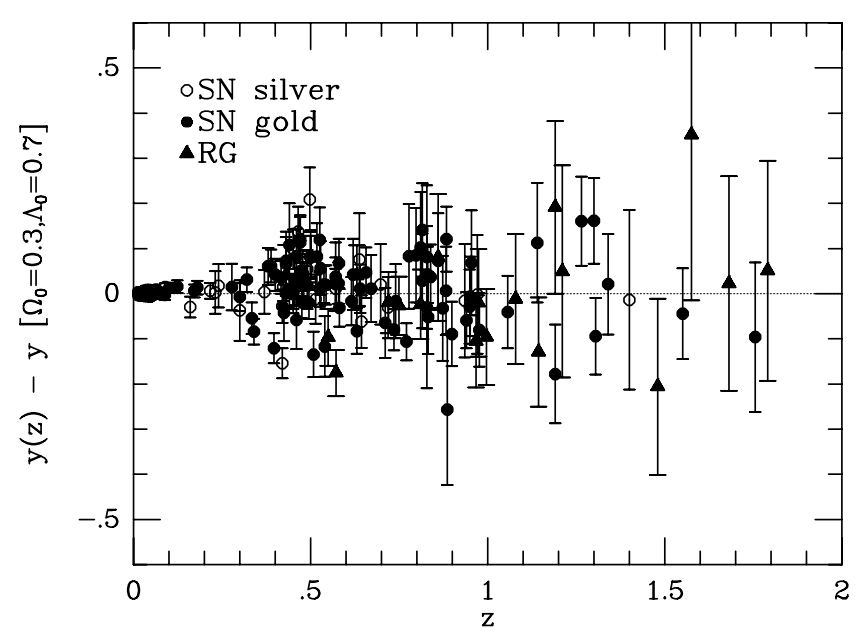

FIG. 1.-Difference between the dimensionless coordinate distances and those expected in a spatially flat universe with a cosmological constant and $\Omega_{0}=0.3$. SNe and RGs are plotted with different symbols as indicated. There is no significant systematic offset between them in the redshift range where there is an overlap.

Figure 3 shows the data from the combined $\mathrm{RG}+\mathrm{SN}$ (gold) sample and the representative fits for $y(z)$ for window function widths $\Delta z$ of 0.4 and 0.6 . Figure 4 shows the corresponding results for the dimensionless expansion rate $E(z)$. We obtain the present value of $E_{0}=0.97 \pm 0.03$. Both trends, $y(z)$ and $E(z)$, are fully consistent with the standard concordance model, which assumes $w=-1, \Omega_{0}=0.3$, and $\Lambda_{0}=0.7$.

Figure 5 shows the trend for the deceleration parameter $q(z)$. This is an update of our result from Paper I, which we believe was the first direct demonstration of the transition from a decelerating to an accelerating universe. This was subsequently seen by Riess et al. (2004), and is further confirmed here, and by Alam et al. (2004). We see a clear trend of an increase in $q(z)$ with redshift out to $z \sim 0.6$, but the fits become noisy and unreliable beyond that because of the still limited number of data points at higher redshifts. The present value is estimated at $q_{0}=-0.35 \pm 0.15$.

The zero crossing is seen at $z_{T} \approx 0.4$; specifically, for the window function with $\Delta z=0.6$, it is $z_{T}=0.35 \pm 0.07$. While the value of $z_{T}$ does not depend significantly on the value of $\Delta z$ used, the size of the uncertainty does, and we are reluctant to quote one particular case. While the lower limit is relatively robust, the upper bound is very uncertain because of the sparse sampling at higher redshifts. We note that in the simple Friedmann-Lemaître models, $z_{T}=\left(2 \Omega_{\Lambda} / \Omega_{0}\right)^{1 / 3}-1$. For the standard concordance model with $w=-1, \Omega_{0}=0.3$, and $\Omega_{\Lambda}=0.7$, we would expect $z_{T}=0.67$. If $z_{T}=0.35$, then the implied value is $\Omega_{\Lambda}=0.55$ for a $k=0$ model. Indeed, the evaluated trend for $q(z)$ is closer to the $\Omega_{\Lambda}=0.5$ model than to the $\Omega_{\Lambda}=0.7$ case, which seems systematically low at a $1-$ $2 \sigma$ level (statistical errors only). However, given the limitations presented by the available data sample, we are unsure about the significance of this effect.

For the subsequent measurements, the assumption that general relativity is the correct theory of gravity is made (see the previous section).

Equation (6) is used to obtain the pressure of the dark energy as a function of redshift, and the results are shown in Figure 6 for a window function with $\Delta z=0.6$. The present value is $p_{0}=-0.6 \pm 0.15$. The results are consistent with the pressure remaining constant to $z \sim 0.5$ and possibly beyond; the strong 

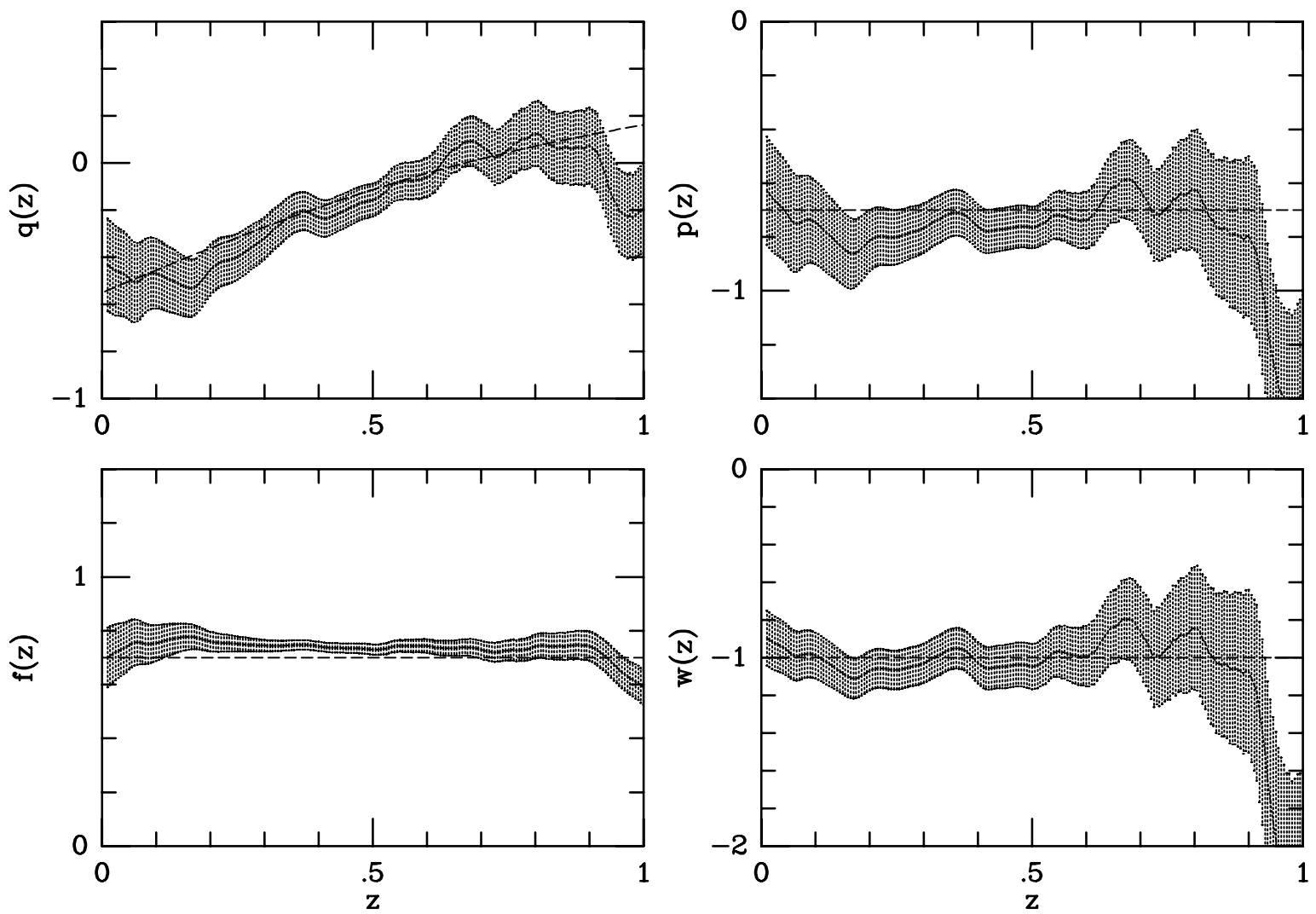

FIG. 2.-Application of our methods to the simulated (pseudo-SNAP) data set, obtained with equations (2), (6), (7), and (8), respectively, as described in the text, using a window function with $\Delta z=0.4$. The hatched regions show the recovered trends for the quantities of interest. The assumed cosmology is a standard Friedmann-Lemaitre model with $\Omega_{0}=0.3$ and $\Lambda_{0}=0.7$, and the theoretical (noiseless) values of the measured quantities are shown as dashed lines. There is a good correspondence (typically well within $\pm 1 \sigma$ ) up to $z \sim 0.9$, except in the case of $f(z)$, where a small systematic bias is present and the formally evaluated errors may be too small as an artifact of the numerical procedure.

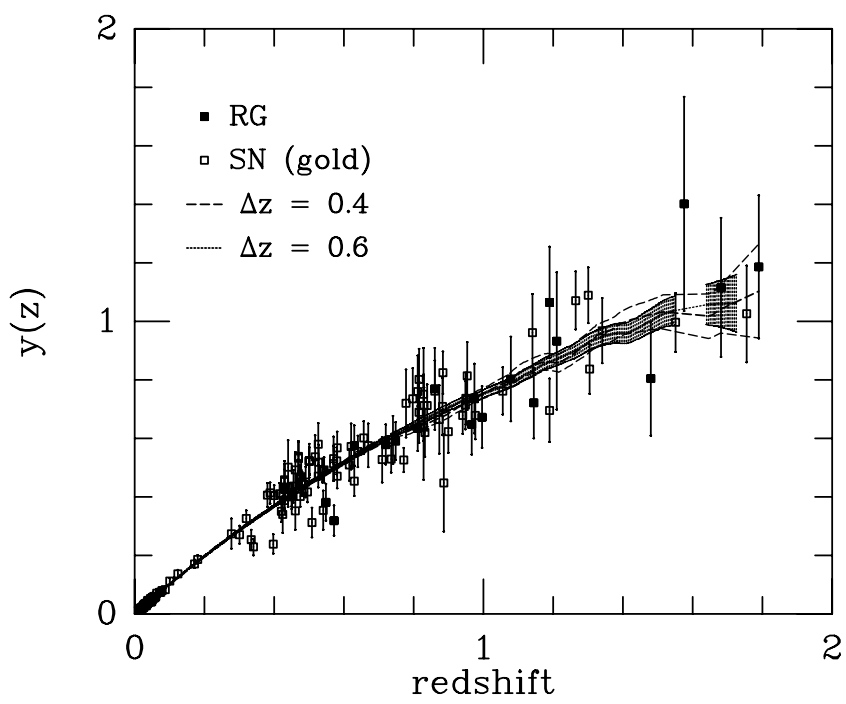

FIG. 3.-Dimensionless coordinate distances $y(z)$ to $20 \mathrm{RGs}$ and the gold sample $\mathrm{SNe}$ as a function of $z$. The smoothed values of $y$, along with their $1 \sigma$ error bars obtained for window function widths $\Delta z=0.4$ (dashed lines) and 0.6 (dotted line and hatched error range) are also shown. Note again that the new high-redshift $\mathrm{SN}$ values agree quite well with those of the highredshift RGs.

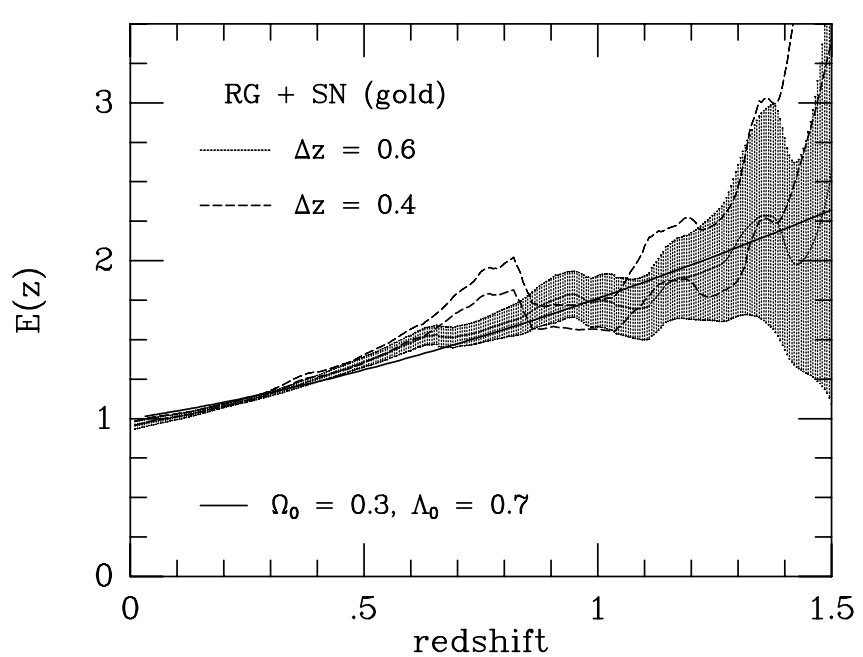

FIG. 4.-Derived values of the dimensionless expansion rate $E(z) \equiv$ $(\dot{a} / a) H_{0}^{-1}=(d y / d z)^{-1}$ obtained with window functions of width $\Delta z=0.4$ and their $1 \sigma$ error bars (dashed lines) and 0.6 (dotted line and hatched error range). At a redshift of zero, the value of $E$ is $E_{0}=0.97 \pm 0.03$. The value of $E(z)$ predicted in a spatially flat universe with a cosmological constant and $\Omega_{0}=0.3$ is also shown and provides a reasonable match to the data. 


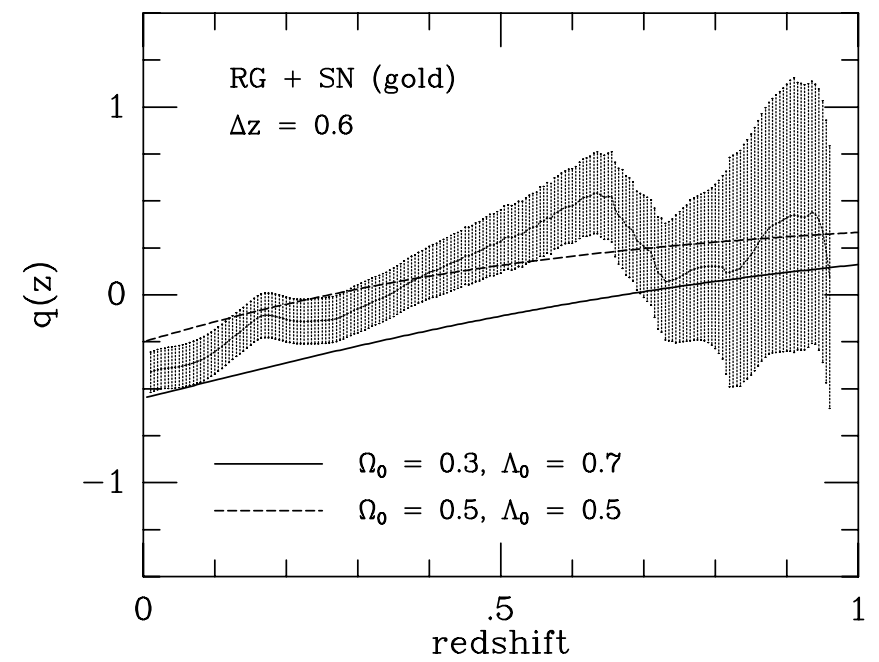

Fig. 5.-Derived values of deceleration parameter $q(z)$ (see eq. [2]) and their $1 \sigma$ error bars obtained with window function of width $\Delta z=0.6$ applied to the RG plus gold SN sample. The universe transitions from acceleration to deceleration at a redshift $z_{T} \approx 0.4$. The value of the deceleration parameter at zero redshift is $q_{0}=-0.35 \pm 0.15$. Note that this determination of $q(z)$ only depends upon the assumptions that the universe is homogenous, isotropic, expanding, and spatially flat, and that it does not depend on any assumptions about the nature of the dark energy or the correct theory of gravity. The solid and dashed lines show the expected dependence in the standard Friedmann-Lemaître models with zero curvature for two pairs of values of $\Omega_{0}$ and $\Lambda_{0}$.

fluctuations at higher redshifts, due to a sparser sampling of data, preclude any stronger statements at this point.

Note that $\rho_{\mathrm{DE} 0}=P_{\mathrm{DE} 0} / w_{0}$, so the value of $p_{0}$ can be used to determine $w_{0}$ if $\rho_{\mathrm{DE} 0}$ is known, or vise versa; for $\Omega_{\mathrm{DE} 0}=$ 0.7 , our determination of $p_{0}$ implies $w_{0}=-0.86 \pm 0.21$, or for $w_{0}=-1$, our determination of $p_{0}$ implies $\Omega_{\mathrm{DE} 0}=0.6 \pm$ 0.15 , which is fully consistent with other measurements of the cosmological constant and our own estimate from the $z_{T}$ given above.

Equation (7) is used to obtain the energy density of the dark energy as a function of redshift, as shown in Figure 7 for the window function width $\Delta z=0.6$, assuming that the mean

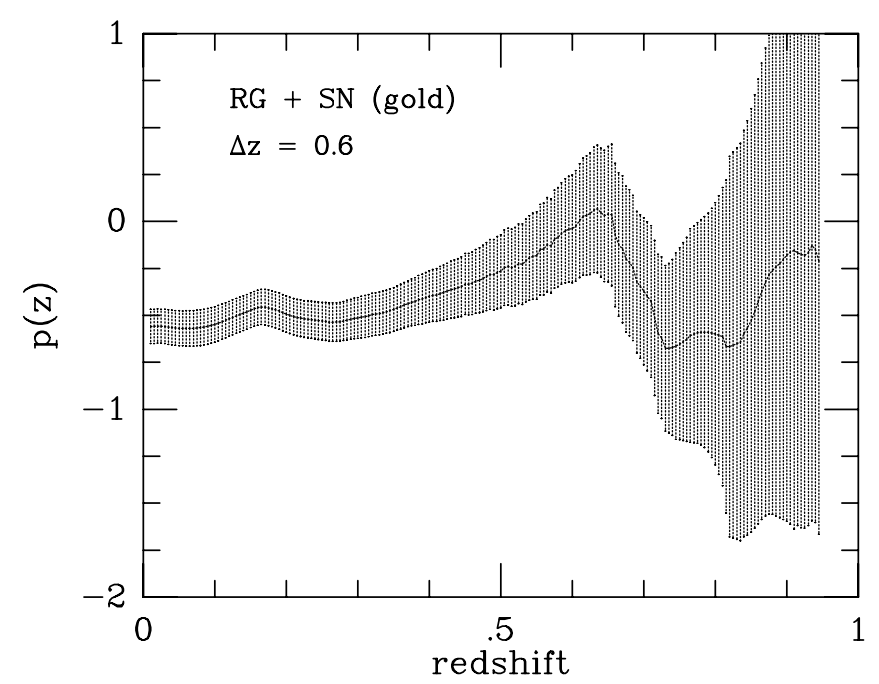

FIG. 6.-Derived values of dark energy pressure $p(z)$ (see eq. [6]), obtained with window function of width $\Delta z=0.6$. This derivation of $p(z)$ requires a choice of theory of gravity, and general relativity has been adopted here. The value at zero redshift is $p_{0}=-0.6 \pm 0.15$.

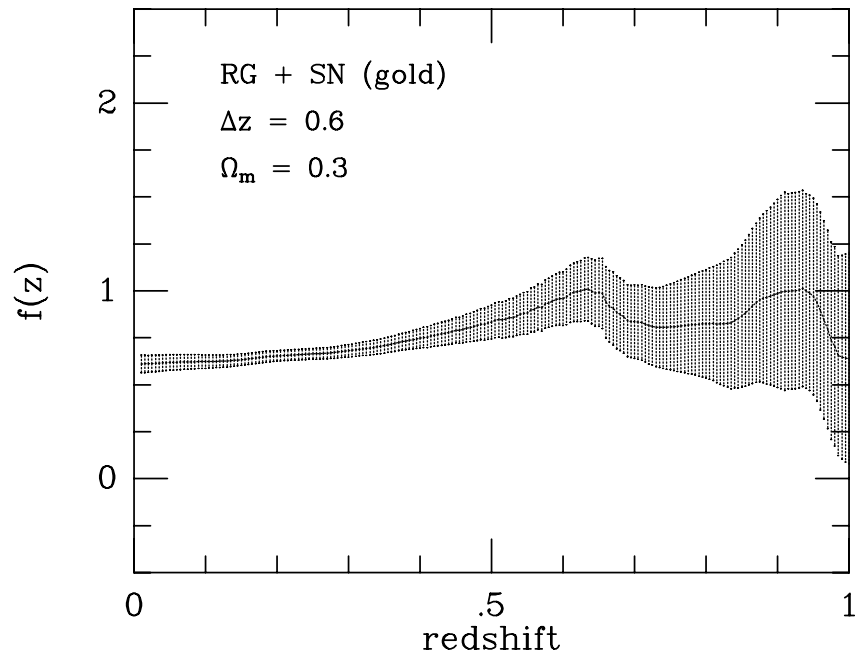

FIG. 7.-Derived values of the dark energy density fraction $f(z)$ (see eq. [7]), obtained with window function of width $\Delta z=0.6$. This derivation of $f(z)$ requires a choice of theory of gravity and the value of $\Omega_{0}$ for the nonrelativistic matter; general relativity has been adopted here, and $\Omega_{0}=0.3$ is assumed. The value at zero redshift is $0.62 \pm 0.05$.

mass density in nonrelativistic matter at zero redshift is $\Omega_{0}=$ 0.3 (implementing different choices for $\Omega_{0}$ is trivial). The present value is $f(z)=0.62 \pm 0.05$. The data are consistent with constant mean dark energy density out to $z \sim 0.5$ and possibly beyond.

Equation (8) is used to study the equation-of-state parameter $w(z)$ as a function of redshift, and the results are shown in Figure 8 . The present value $w_{0}=-0.9 \pm 0.1$ is fully consistent with the interpretation of the dark energy as a cosmological constant $(w=-1)$. However, the trend out to $z \sim 0.6$ is intriguing. We are uncertain at this point whether this is simply due to a sampling-induced fluctuation (as is obviously the case at higher redshifts), or whether there may be a real evolution of $w(z)$. Clearly, to invoke the standard cosmological truism, more data are needed.

In all of our analyses, we have also considered different samples and subsamples of data, such as including a sample of

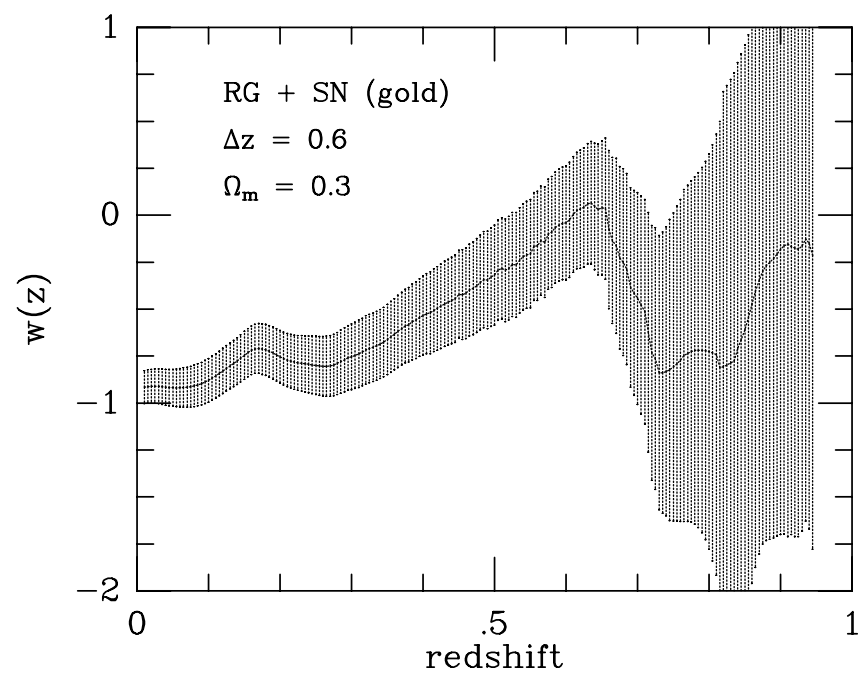

FIG. 8. -Derived values of the dark energy equation-of-state parameter $w(z)$ (see eq. [8]), obtained with window function of width $\Delta z=0.6$. This derivation of $w(z)$ requires a choice of theory of gravity and the value of $\Omega_{0}$; general relativity has been adopted here, and $\Omega_{0}=0.3$ is assumed. The value at zero redshift is $w_{0}=-0.9 \pm 0.1$, consistent with the cosmological-constant models. 
just the silver and gold SNe (Riess et al. 2004), the gold SNe alone, and the sample of RGs plus silver and gold SNe; the results are effectively the same as those shown here. However, we note that since the $\mathrm{SNe}$ dominate the joint sample, all of our results are just as vulnerable to any hidden systematic errors that may be present in the data as the more traditional analysis presented by Riess et al.

\section{SUMMARY}

We have expanded and used the method developed in Paper I on a new sample of coordinate distances to $\mathrm{SNe}$ and RGs to evaluate the trends of the expansion rate $E(z)$, deceleration parameter $q(z)$, pressure of the dark energy $p(z)$, its fractional energy density $f(z)$, and its equation-of-state parameter $w(z)$ as functions of redshift. We make an assumption that the FRW metric is valid, and we make the observationally supported assumption of the spatially flat universe. This enables us to derive the trends for $E(z)$ and $q(z)$, which are otherwise model-independent, and thus can help discriminate at least some proposed models of the dark energy. By assuming that the standard general relativity is the correct theory of gravity on cosmological scales, we can also produce trends of $p(z), f(z)$, and $w(z)$ without any additional assumptions about the nature of dark energy. These trends may also be used to discriminate between different physical models of the dark energy.

We find that the data are generally but perhaps not entirely consistent with the standard Friedmann-Lemaitre concordance cosmology with $w=-1, \Omega_{0}=0.3$, and $\Lambda_{0}=0.7$, although somewhat lower values of $\Lambda_{0}$ may be preferred.

We confirm the result Paper I and that of Riess et al. (2004) that there is a clear increase in $q(z)$ with redshift, with the present value $q_{0}=0.35 \pm 0.1$ and the transition from decelerating to accelerating universe at $z_{T} \approx 0.4$.

Functions $p(z), f(z)$, and $w(z)$ are consistent with being constant at least out to $z \sim 0.5$ and possibly beyond; the existing data are inadequate to constrain their evolution beyond $z \sim 0.5$, but there are some hints of increase with redshift for $f(z)$ and $w(z)$.

At lower redshifts, the data are consistent with cosmologicalconstant models. We obtain for the present values $w_{0}=-0.9 \pm$ 0.1 and $p_{0}=-0.6 \pm 0.15\left(=-\Lambda_{0}\right.$ for the Friedmann-Lemaître models).

Even with the currently available data, these results represent new observational constraints for models of the dark energy. We believe that this methodology will prove increasingly useful in determining the nature and evolution of the dark energy as better and more extensive data sets become available. Clearly, this method works best when redshift space is densely sampled. Our current results suggest that redshift space is sufficiently sampled at redshifts less than about 0.4 . More accurate results could be obtained by increasing the sampling of data points with redshifts greater than 0.4 , particularly in the redshift range from 0.4 to 1.5 .

It is a pleasure to thank Megan Donahue, Chris O'Dea, Saul Perlmutter, and Adam Riess for helpful discussions. This work was supported in part by the U. S. National Science Foundation under grant AST 02-06002 and by Penn State University (R. A. D.) and the Ajax Foundation (S. G. D.). Finally, we acknowledge the great work and efforts of many observers who obtained the valuable data used in this study.
Alam, U., Sahni, V., Saini, T. D., \& Starobinsky, A. A. 2003, MNRAS, 344,1057

Alam, U., Sahni, V., \& Starobinsky, A. 2004, J. Cosmology Astropart. Phys., 6,8

Astier, P. 2001, Phys. Lett. B, 500, 8

Barger, V., \& Marfatia, D. 2001, Phys. Lett. B, 498, 67

Barris, B. J., et al. 2004, ApJ, 602, 571

Bennett, C., et al. 2003, ApJ, 583, 1

Chiba, T., \& Nakamura, T. 2000, Phys. Rev. D, 62, 121301

Daly, R. A., \& Djorgovski, S. 2003, ApJ, 597, 9 (Paper I) 2004, preprint (astro-ph/0405063)

Daly, R. A., \& Guerra, E. J. 2002, AJ, 124, 1831

Elgaroy, O., \& Multamaki, T. 2004, preprint (astro-ph/0404402)

Gerke, B. F., \& Efstathiou, G. 2002, MNRAS, 335, 33

Goliath, M., Amanullah, T., Astier, P., Goobar, A., \& Pain, R. 2001, A\&A, 380,6

Gong, Y. 2004, preprint (astro-ph/0401207)

Guerra, E. J., \& Daly, R. A. 1998, ApJ, 493, 536

Guerra, E. J., Daly, R. A., \& Wan, L. 2000, ApJ, 544, 659

Huterer, D., \& Cooray, A. 2004, preprint (astro-ph/0404062)

Huterer, D., \& Starkman, G. 2003, Phys. Rev. Lett., 90, 031301

Huterer, D., \& Turner, M. S. 1999, Phys. Rev. D, 60, 081301 . 2001, Phys. Rev. D, 64, 123527

Knop, R. A., et al. 2003, ApJ, 598, 102

Maor, I., Brustein, R., \& Steinhardt, P. 2001, Phys. Rev. Lett., 86, 6

\section{REFERENCES}

Nessier, S., \& Perivolaropoulos, L. 2004, preprint (astro-ph/0401556)

Padmanabhan, T., \& Choudhury, T. R. 2003, MNRAS, 344, 823

Peebles, P. J. E. 1993, Principles of Physical Cosmology (Princeton: Princeton Univ. Press)

Peebles, P. J. E., \& Ratra, B. 2003, Rev. Mod. Phys., 75, 559

Perlmutter, S., et al. 1999, ApJ, 517, 565

Podariu, S., Daly, R. A., Mory, M. P., \& Ratra, B. 2003, ApJ, 584, 577

Riess, A. G., et al. 1998, AJ, 116, 1009 2004, ApJ, 607, 665

Sahni, V., Saini, T. D., Starobinsky, A. A., \& Alam, U. 2003, J. Exp. Theor. Phys. Lett., 77, 201

Saini, T., Raychaudhury, S., Sahni, V., \& Starobinsky, A. A. 2000, Phys. Rev. Lett., 85, 1162

Spergel, D., et al. 2003, ApJS, 148, 175

Starobinsky, A. 1988, Soviet Phys.-JETP Lett., 68, 757

Tegmark, M. 2002, Phys. Rev. D, 66, 103507

Tonry, J. T., et al. 2003, ApJ, 594, 1

Wang, Y., \& Freese, K. 2004, preprint (astro-ph/0402208)

Wang, Y., \& Garnavich, P. 2001, ApJ, 552, 445

Wang, Y., Kostov, V., Freese, K., Frieman, J. A., \& Gondolo, P. 2004, Phys. Rev. D., submitted (astro-ph/0402080)

Wang, Y., \& Tegmark, M. 2004, Phys. Rev. Lett., 92, 241302

Weinberg, S. 1972, Gravitation and Cosmology (New York: Wiley)

Weller, J., \& Albrecht, A. 2002, Phys. Rev. D, 65, 103512

Zhu, Z.-H., Fujimoto, M., \& He, X.-T. 2004, ApJ, 603, 365 